\title{
Nocturnal oximetry for the diagnosis of the sleep apnoea hypopnoea syndrome: a method to reduce the number of polysomnographies?
}

\author{
Eusebi Chiner, Jaime Signes-Costa, Juan Manuel Arriero, Juan Marco, Isabel Fuentes, \\ Antonia Sergado
}

\begin{abstract}
Background-Polysomnography (PSG) is currently the "gold standard" for the diagnosis of the sleep apnoea hypopnoea syndrome (SAHS). Nocturnal oximetry (NO) has been used with contradictory results. A prospective study was performed to determine the accuracy of NO as a diagnostic tool and to evaluate the reduction in the number of PSGs if the diagnosis of SAHS had been established by this method.

Methods-Two hundred and seventy five patients with a clinical suspicion of SAHS were admitted to undergo, in the same night, full PSG and NO. Desaturation was defined as a fall in the haemoglobin saturation level $\left(\mathrm{SaO}_{2}\right)$ to lower than $4 \%$ from the baseline level and an oxygen desaturation index per hour (ODI) was obtained in each patient with three cut off points: $\geqslant 5$ (ODI-5), $\geqslant 10$ (ODI-10), and $\geqslant 15$ (ODI-15).
\end{abstract}

Results-SAHS was diagnosed in 216 patients (194 men). After withdrawing patients with abnormal lung function (forced expiratory volume in one second $\left(F_{1} V_{1}\right)$ lower than $80 \%$ predicted), sensitivity (SE), specificity (SP), positive and negative predictive values (PPV and NPV) of NO were: ODI-5 $(80 \%, 89 \%, 97 \%, 48 \%)$; ODI-10 (71\%, 93\%, 97\%, 42\%); ODI-15 $(63 \%, 96 \%, 99 \%, 38 \%)$. The accuracy for each ODI was $0.81,0.75$, and 0.70 , respectively. If NO had been considered as a diagnostic tool and PSG had been performed only in patients with a negative NO (false negative and true negative) and those with a positive NO and abnormal pulmonary function tests, 135/275 (ODI5), 156/275 (ODI-10), and 170/275 (ODI15) PSGs would have been performed, a reduction of 140,119 , and 105 , respectively.

Conclusion-Nocturnal oximetry in patients with suspected SAHS and normal spirometric values permits the institution of therapeutic measures in most patients. (Thorax 1999;54:968-971)

Keywords: sleep apnoea hypopnoea syndrome; sleep apnoea syndrome; nocturnal oximetry; diagnosis

Sleep apnoea hypopnoea syndrome (SAHS) is a major health problem due to its prevalence rates in middle aged people of $2-4 \% .{ }^{1}$ Signifi- cant morbidity ${ }^{2}$ and mortality ${ }^{3}$ have been reported in patients with SAHS. Full polysomnography (PSG) is currently the gold standard test in the diagnosis of SAHS. However, strategies to confirm or refute a diagnosis of SAHS differ from one country to another. While some state that PSG should be performed in all patients with a clinical suspicion of SAHS, ${ }^{45}$ others recommend less expensive and time consuming tests such as nocturnal oximetry (NO). ${ }^{6}$

NO has been evaluated in patients with SAHS because it analyses arterial oxygen desaturation, one of the consequences of SAHS. However, contradictory results have been obtained in different studies ${ }^{7-10}$ and controversy exists about its use as a case selection tool in SAHS. In Spain and most European countries with universal health coverage a large number of patients requiring assistance in sleep disorders clinics must be properly diagnosed and managed with limited resources. ${ }^{11}$ We have designed a prospective study to analyse the accuracy of $\mathrm{NO}$ as a diagnostic tool in patients suspected of having SAHS and to evaluate the number of PSGs that could have been saved if the diagnosis of SAHS had been established by this method.

\section{Methods}

PATIENTS

Over a two year period 275 consecutive patients were prospectively studied in our sleep respiratory disorders unit which is located in a 400 bed university hospital. Our institution takes referrals for sleep disorders from a population of 1200000 in Alicante (Spain). Patients were referred to our unit by pulmonary physicians (60\%), neurologists $(20 \%)$, general practitioners $(20 \%)$, and others $(10 \%)$.

PROTOCOL

All patients with a clinical suspicion of SAHS - that is, one or more of (a) daytime sleepiness, (b) loud snoring, and (c) reported interrupted nocturnal breathing - were seen in our sleep outpatient clinic and then, without any exclusion criteria, they were included in a protocol with a physical examination, blood tests, chest radiography, arterial blood gas tensions, pulmonary function tests, electrocardiography, and measurement of daytime sleepiness with the Epworth sleepiness scale. ${ }^{12}$ Oropharyngeal and craniofacial abnormalities were classified by inspection as normal or abnormal and those in the latter category were 
Table 1 Mean (SD) demographic data and comparisons between groups

\begin{tabular}{llll}
\hline & Non-SAHS & SAHS & p value \\
\hline Men (n) & 52 & 194 & NS \\
Women (n) & 7 & 22 & NS \\
Age (years) & $48(14)$ & $53(10)$ & $<0.01$ \\
Body mass index $\left(\mathrm{kg} / \mathrm{m}^{2}\right)$ & $30(5)$ & $32(5)$ & $<0.01$ \\
Epworth sleepiness scale & $10(5)$ & $14(5)$ & $<0.001$ \\
Neck circumference $(\mathrm{cm})$ & $43(4)$ & $43(4)$ & NS \\
\hline
\end{tabular}

referred for more detailed examination. Patients were then admitted to our unit to undergo a nocturnal full PSG and oximetry.

OXIMETRY

A pulse oximeter (N-200; Nellcor Inc, Hayward, California, USA) was attached to the subject's finger using a flexible probe. The instrument detects 10 data points per minute, each point representing the lowest saturation in a six second interval. A desaturation event was considered when the haemoglobin saturation level $\left(\mathrm{SaO}_{2}\right)$ fell below $4 \%$ from baseline saturation. Baseline saturation was considered as the mean saturation in the previous minute. Falls in oxygen saturation to $\geqslant 4 \%$ in the interval $90-100 \%$ of saturation were also considered as desaturations. Oximetry values were periodically checked using arterial blood gas samples. The signals were digitalised and recorded using the package software, and were manually reviewed by two observers blinded to the polysomnographic data. The total number of

Table 2 Sensitivity (SE), specificity (SP), positive predictive value (PPV) and negative predictive value (NPV) and 95\% confidence interval (CI) of nocturnal oximetry for each cut off point in 216 patients with SAHS (194 men, 22 women)

\begin{tabular}{lllll}
\hline & $S E \%(C I)$ & $S P \%(C I)$ & $P P V \%(C I)$ & NPV\% (CI) \\
\hline ODI $\geqslant 5$ & $82(77$ to 87$)$ & $76(63$ to 86$)$ & $93(88$ to 96$)$ & $54(43$ to 65$)$ \\
ODI $\geqslant 10$ & $71(65$ to 77$)$ & $90(79$ to 96$)$ & $96(92$ to 99$)$ & $46(37$ to 56$)$ \\
ODI $\geqslant 15$ & $62(56$ to 69$)$ & $93(83$ to 98$)$ & $97(92$ to 99$)$ & $40(32$ to 49$)$ \\
\hline
\end{tabular}

Table 3 Comparison between false negative (FN) and true positive (TP) patients when cut off points in the oxygen desaturation index (ODI) of $\geqslant 5, \geqslant 10$, and $\geqslant 15$ were considered to analyse the nocturnal oximetry

\begin{tabular}{|c|c|c|c|}
\hline & $F N$ & $T P$ & p value \\
\hline \multicolumn{4}{|l|}{$O D I \geqslant 5$} \\
\hline Age (years) & $51(10)$ & $53(10)$ & NS \\
\hline Duration of symptoms (months) & $53(52)$ & $72(72)$ & 0.053 \\
\hline $\mathrm{BMI}\left(\mathrm{kg} / \mathrm{m}^{2}\right)$ & $29(4)$ & $33(5)$ & $<0.001$ \\
\hline Epworth sleepiness scale & $12(4)$ & $14(5)$ & $<0.01$ \\
\hline Neck circumference $(\mathrm{cm})$ & $41(4)$ & $44(4)$ & $<0.01$ \\
\hline Cephalometric abnormalities & $6 / 37$ & $56 / 175$ & 0.08 \\
\hline AHI $(\mathrm{n})$ & $22(7)$ & $46(20)$ & $<0.001$ \\
\hline Baseline $\mathrm{SaO}_{2}(\mathrm{kPa})$ & $12.5(0.3)$ & $12.1(0.4)$ & $<0.001$ \\
\hline Minimal $\mathrm{SaO}_{2}(\mathrm{kPa})$ & $11.6(0.7)$ & $9.4(1.6)$ & $<0.001$ \\
\hline \multicolumn{4}{|l|}{$O D I \geqslant 10$} \\
\hline Age (years) & $53(10)$ & $53(10)$ & NS \\
\hline Duration of symptoms (months) & $52(52)$ & $74(74)$ & $<0.01$ \\
\hline $\mathrm{BMI}\left(\mathrm{kg} / \mathrm{m}^{2}\right)$ & $29(4)$ & $33(6)$ & $<0.001$ \\
\hline Epworth sleepiness scale & $12(5)$ & $15(5)$ & $<0.001$ \\
\hline Neck circumference $(\mathrm{cm})$ & $42(3)$ & $44(4)$ & $<0.01$ \\
\hline Cephalometric abnormalities & $11 / 61$ & $52 / 152$ & $<0.05$ \\
\hline $\mathrm{AHI}(\mathrm{n})$ & $24(11)$ & $49(19)$ & $<0.001$ \\
\hline Baseline $\mathrm{SaO}_{2}(\mathrm{kPa})$ & $12.4(0.4)$ & $12.1(0.4)$ & $<0.01$ \\
\hline Minimal $\mathrm{SaO}_{2}(\mathrm{kPa})$ & $11.2(0.9)$ & $9.6(1.6)$ & $<0.001$ \\
\hline \multicolumn{4}{|l|}{$O D I \geqslant 15$} \\
\hline Age (years) & $52(10)$ & $53(10)$ & NS \\
\hline Duration of symptoms (months) & $49(49)$ & $65(65)$ & $<0.05$ \\
\hline $\mathrm{BMI}\left(\mathrm{kg} / \mathrm{m}^{2}\right)$ & $30(4)$ & $33(5)$ & $<0.001$ \\
\hline Epworth sleepiness scale & $12(5)$ & $15(5)$ & $<0.001$ \\
\hline Neck circumference $(\mathrm{cm})$ & $42(3)$ & $44(4)$ & $<0.001$ \\
\hline Cephalometric abnormalities & $15 / 80$ & $47 / 133$ & $<0.05$ \\
\hline $\mathrm{AHI}(\mathrm{n})$ & $26(12)$ & $51(18)$ & $<0.001$ \\
\hline Baseline $\mathrm{SaO}_{2}(\mathrm{kPa})$ & $12.4(0.4)$ & $12.1(0.4)$ & $<0.01$ \\
\hline Minimal $\mathrm{SaO}_{2}(\mathrm{kPa})$ & $11(0.9)$ & $9.3(1.6)$ & $<0.001$ \\
\hline
\end{tabular}

$\mathrm{BMI}=$ body mass index; $\mathrm{AHI}=$ apnoea hypopnoea index; $\mathrm{SaO}_{2}=$ oxygen saturation. desaturations was divided by the hours in bed and an oxygen desaturation index per hour (ODI) was obtained for each patient with three cut off points: $\geqslant 5$ (ODI-5), $\geqslant 10$ (ODI-10), and $\geqslant 15$ (ODI-15).

POLYSOMNOGRAPHY

Patients underwent standard overnight polysomnography (Somnostar $\alpha$ 4100; Sensor Medics, California, USA) which included continuous recordings of electroencephalography (EEG), electro-oculography (EOG), and chin electromyography (EMG) for sleep staging according to the criteria of Rechtshaffen and Kales. ${ }^{13}$ Nasal and oral thermistors were used to measure airflow and uncalibrated inductance plethysmography was used to measure rib cage and abdominal motion. The electrocardiogram (ECG) and $\mathrm{SaO}_{2}$ were also continuously monitored. Apnoea was defined as a cessation of flow of more than 10 seconds duration. Hypopnoea was defined as a $50 \%$ reduction in the flow signal or thoracoabdominal movement compared with the baseline value of immediately preceding breaths lasting more than 10 seconds accompanied by a fall of $\geqslant 4 \%$ in saturation or a microarousal. A desaturation was therefore not a requisite criterion if a microarousal was present with reduction in airflow. SAHS was defined as an apnoea plus hypopnoea index (AHI) of 15 or more. ${ }^{14}$ All signals were computer analysed with manual editing.

DATA ANALYSIS

Data from NO and PSG were stored in a database for statistical analysis (Rsigma, Horus, Madrid, Spain) and were independently analysed using NO as the test and PSG as the gold standard for the correct classification of SAHS patients. The number of true positive (TP), false positive (FP), false negative $(\mathrm{FN})$, and true negative (TN) oximetric results were then determined. Sensitivity $(\mathrm{TP} / \mathrm{TP}+\mathrm{FN})$, specificity $(\mathrm{TN} / \mathrm{TN}+\mathrm{FP})$, positive predictive values $(\mathrm{TP} / \mathrm{TP}+\mathrm{FP})$, and negative predictive values (TN/TN+FN) and confidence intervals (CI) were calculated for each ODI. The accuracy $(\mathrm{TP}+\mathrm{TN} / \mathrm{FP}+\mathrm{FN}+\mathrm{TN}+\mathrm{TP})$ of $\mathrm{NO}$ was also obtained for each cut off point and then recalculated for each ODI after the withdrawal of patients with abnormal pulmonary function $\left(\mathrm{FEV}_{1}\right.$ lower than $80 \%$ predicted $\left.^{15}\right)$. Numerical data were expressed as mean values and standard deviation (SD). Numerical variables between groups were compared using the Student's $t$ test for unpaired data and the Mann-Whitney test for variables not normally distributed. Qualitative data were compared using the $\chi$ and Fisher exact tests. A $\mathrm{p}$ value of $<0.05$ was considered significant.

\section{Results}

A total of 275 patients were recruited to the study and 216 were diagnosed with SAHS. The mean (SD) AHI was 42 (20), range 15-101. No differences in sex were observed between the SAHS and non-SAHS groups. Patients with SAHS were older $(p<0.01)$, more obese 
Table 4 Sensitivity (SE), specificity (SP), positive predictive value (PPV) and negative predictive value (NPV) and 95\% confidence interval (CI) of nocturnal oximetry for each cut off point in 216 patients with SAHS after the withdrawal of patients with abnormal spirometric values

\begin{tabular}{lllll}
\hline & $S E \%(C I)$ & $S P \%(C I)$ & $P P V \%(C I)$ & $N P V \%$ (CI) \\
\hline ODI $\geqslant 5$ & $80(71$ to 86$)$ & $89(69$ to 97$)$ & $97(91$ to 99$)$ & $48(34$ to 63$)$ \\
ODI $\geqslant 10$ & $71(62$ to 79$)$ & $93(74$ to 99$)$ & $97(91$ to 99$)$ & $42(29$ to 55$)$ \\
ODI $\geqslant 15$ & $63(54$ to 72$)$ & $96(78$ to 100$)$ & $99(92$ to 100$)$ & $38(27$ to 51$)$ \\
\hline
\end{tabular}

$(\mathrm{p}<0.01)$, and had more daytime sleepiness $(\mathrm{p}<0.001)$ (table 1$)$.

When a diagnosis of SAHS was established by PSG the results of NO at the different cut off points were analysed. With ODI-5 192 patients had positive oximetry results (178 TP and $14 \mathrm{FP}$ ), with ODI-10 there were 160 patients with a positive oximetry test $(154 \mathrm{TP}$ and six FP), and with ODI-15 139 patients had positive oximetry results $(135 \mathrm{TP}$ and four FP). The accuracy for each ODI was 0.81 , 0.75 , and 0.69 , respectively. The sensitivity (SE), specificity (SP), positive predictive values (PPV), and negative predictive values (PPV) are shown for each cut off point in table 2 .

When the FN patients in each ODI group were analysed they were found to have a lower $\mathrm{AHI}$, higher baseline and minimal saturation, a shorter time with symptoms, less daytime sleepiness, lower body mass index, and fewer craniofacial abnormalities than TP patients, but no differences were found in age or pulmonary function tests between these two groups of patients (table 3)

Patients with abnormal oximetry (ODI-5) and AHI of $<15$ (FP) had the following diagnoses: chronic obstructive pulmonary disease (COPD) in eight, obesity hypoventilation in three, ischaemic heart disease in two, and one with myotonic dystrophia. These patients had poorer lung function than TP patients (forced vital capacity (FVC) 74 (18)\% vs 87 (17) \%, p <0.01; $\mathrm{FEV}_{1} 60$ (23)\% vs $83(22) \%$, $\mathrm{p}<0.01$; and $\mathrm{FEV}_{1} / \mathrm{FVC} 78$ (23)\% vs $91(17) \%$, $\mathrm{p}<0.01)$. The SE, SP, PPV and NPV of NO after withdrawal of patients with abnormal spirometric values are shown for each cut off point in table 4 . The accuracy for each ODI was $0.81,0.75$, and 0.70 , respectively.

If we had considered NO as a diagnostic tool in the patients with SAHS included in our study and had performed PSG only in those with a negative NO (FN and TN) or those with a positive $\mathrm{NO}$ and abnormal pulmonary function tests, we would have performed 135/275 PSGs for a cut off point of ODI-5, 156/275 for ODI-10, and 170/275 for ODI-15, reducing the number of PSGs by 140,119 , and 105 , respectively.

\section{Discussion}

This study shows that a quantitative analysis of pulse oximetry can help in the diagnosis of SAHS and this approach can reduce the number of PSGs in countries with limited resources. The sensitivity of $\mathrm{NO}$ at different cut off points ranged between $82 \%$ (ODI-5) and $62 \%$ (ODI-15), whilst specificity varied between $76 \%$ (ODI-5) and 93\% (ODI-15). The accuracy for each ODI was $0.81,0.75$, and 0.69 , respectively, which did not change $(0.81$,
0.75 , and 0.70$)$ after withdrawal of patients with abnormal spirometric values.

These results differ from those of other studies. ${ }^{7-10}$ Several possible reasons can be put forward to explain these discrepancies. When the population studied has a high prevalence of SAHS the sensitivity and specificity of NO may improve because the accuracy of NO lies in the detection of apnoeas while hypopnoeas are underdiagnosed. In this study 216 of 275 patients had a diagnosis of SAHS by PSG, a prevalence of $78 \%$ which is higher than that in any of the previous studies. ${ }^{7-10}$ Sleep units in countries with universal health coverage have a bias towards studying patients who are more likely to have positive tests (patients with a high clinical suspicion of SAHS). Moreover, because our unit has a specific profile in respiratory sleep disorders and $60 \%$ of referrals were made by pulmonary physicians, this high prevalence may be justified.

Another explanation for the disparity of the results is the method of quantifying nocturnal desaturation. ${ }^{9}$ Oximeters have different time responses and, depending on the settings, they can underestimate the number of desaturations. Modifying the average time of the pulse oximeter would account for up to $60 \%$ of underestimated desaturations. ${ }^{16}$

Pépin et al used a mathematical index to detect changes in $\mathrm{SaO}_{2}$ associated with sleep apnoeas with a sensitivity of $75 \%$ and a specificity of $86 \% \cdot{ }^{17}$ In spite of a small number of patients with different disorders (SAHS, COPD, and restrictive lung disease), their results do not differ significantly from ours when using a point intermediate between ODI-5 and ODI- 10.

Williams et al used a visual analysis of oximeter tracings without taking into account desaturations of $\geqslant 4 \%$ when they occurred at levels of saturation above $90 \%$. They obtained a sensitivity of $75 \%$ and a specificity of $100 \%$ in a small number of patients.

Sériès $e t a l^{18}$ used oximetry as a diagnostic tool but the high numbers of false positives negated its use as a case selection tool for detecting SAHS in suspected patients. They did not define desaturations with a fixed numerical criterion, counting as abnormal patients who had more than 10 transient desaturations per hour followed by a rapid return to the baseline level. Moreover, they did not show pulmonary function data in patients with normal polysomnography and more than 10 desaturations per hour.

Gyulay et $a l^{19}$ identified patients with SAHS using clinical assessment and home oximetry. More than 15 desaturations/hour of $\geqslant 4 \%$ gave a sensitivity of $40 \%$ and a specificity of $98 \%$. They concluded that patients with positive oximetry should start on treatment with continuous positive airway pressure (CPAP). These results with ODI-15 could be improved if the settings of the oximeter had been put at six seconds as in our study. On the other hand, the population they studied had a low prevalence of SAHS.

Epstein et $a l^{10}$ compared the cost of nocturnal oximetry as a case selection tool with the 
cost of standard PSG for the detection of SAHS. They concluded that the use of $\mathrm{NO}$ as a diagnostic tool was limited because of the high rate of false positive results. Their diagnostic decision trees caused them to conclude that the use of $\mathrm{NO}$ as a case selection tool is not justified on the basis of cost effectiveness but they gave no explanation for the high number of false positives.

In the present study patients with a false positive NO had pulmonary function values significantly lower than the true positive group. Gries et $a l^{20}$ studied $\mathrm{SaO}_{2}$ during sleep in 350 subjects with negative PSG results (AHI <5). Patients with known or suspected respiratory disorders were excluded. They obtained values of the lowest saturation during the night, the saturation below which the patient spent $10 \%$ of the time (Sat 10), and the median saturation (Sat 50). This apparently healthy population did not desaturate during sleep and only subjects over 60 years of age had a significantly lower Sat 50 and Sat 10. Their data are consistent with our results and show that only patients with a pulmonary disorder, mainly COPD, have normal PSG results (AHI $\leqslant 15$ ) and abnormal nocturnal oximetry.

In our study NO misclassified patients (false negatives) with less severe disease as evidenced by a shorter time with symptoms, lower body mass index, less sleepiness, smaller neck circumference, lower AHI, and higher baseline and minimal $\mathrm{SaO}_{2}$. Moreover, hypopnoeas were considered on PSG in the absence of a fall in $\mathrm{SaO}_{2}$ when a microarousal was detected. These patients may also have had shorter apnoeas, being less likely to be followed by a fall in $\mathrm{SaO}_{2}$. Nevertheless, we would not miss these patients if all patients with negative NO underwent full PSG. Some of these patients, probably because of their mild disease, may not receive specific treatment such as nasal CPAP.

In our study the PPV of NO is very high (93\% for ODI-5 to $97 \%$ for ODI-15), allowing us to initiate treatment in patients without respiratory disorders but with abnormal $\mathrm{NO}$, as recommended by the British Thoracic Society. ${ }^{6}$

Some of the criticism of the use of $\mathrm{NO}$ as a diagnostic test rests on the concern that patients may not be sleeping. However, patients without cardiopulmonary disorders do not tend to desaturate. ${ }^{20}$ Moreover, it has been reported that neurophysiological parameters may play a less significant part than respiratory events. ${ }^{21}$

One aim of this study was to determine the number of PSGs that would be saved if we had used NO as a diagnostic test, with full PSG being used only in patients with a negative NO and those with a positive NO and abnormal pulmonary function. With this approach we would have saved 140,119, and 105 PSGs, respectively, at the three different cut off points (ODI-5, ODI-10, and ODI-15). Using higher cut off points (i.e. ODI $\geqslant 15$ ) the PPV of NO reaches almost $100 \%$; however, the decrease in sensitivity would have generated more full PSGs. We did not analyse the cost savings using this approach but if we had used $\mathrm{NO}$ as a first diagnostic test we would have saved a significant number of PSGs.

Our results have been obtained using oximetry in the hospital setting. To our knowledge differences between home and hospital oximetry have not been reported but, if any exist, the probability of achieving a positive result with oximetry would be likely to be even higher at home, making this test more useful.

We conclude that starting treatment in patients with NO results suggestive of SAHS and normal spirometric values is a way of decreasing the need for PSG and of helping to manage these patients clinically. This would be a means of prioritising diagnostic or therapeutic PSG. Meanwhile, it needs to be determined whether effective CPAP titration can be conducted with NO, at least in some patients with SAHS, to help reduce the number of PSGs being performed for CPAP titration.

1 Young T, Palta M, Dempsey J, et al. The occurrence of sleep-disordered breathing among middle-aged adults. $N$ Engl f Med 1993;328:1230-5.

2 Findley LJ, Unverzagt ME, Suratt PM. Automobile accidents involving patients with obstructive sleep apnea. Am Rev Respir Dis 1988;138:337-40.

$3 \mathrm{He} \mathrm{J}$, Kryger MH, Zorick FJ, et al. Mortality and apnea index in obstructive sleep apnea. Experience in 385 male patients. Chest 1988;94:9-14.

4 American Thoracic Society. Indications and standards for cardiopulmonary sleep studies. Am Rev Respir Dis 1989, 139:559-68.

5 Barbé F, Amibilia J, Capote F, et al. Diagnóstico del síndrome de apneas obstructivas durante el sueño. Informe de Consenso del Area de Insuficiencia Respiratoria y Trastornos del Sueño. Arch Bronconeumol 1995;31:460-2.

6 British Thoracic Society. Facilities for the diagnosis and treatment of abnormal breathing during sleep including treatment of abnormal breathing during sleep
nocturnal ventilation. BTS News 1990;5:7-10.

7 Williams AJ, Yu G, Santiago S, et al. Screening for sleep apnea using pulse oximetry and a clinical score. Chest 1991;100:631-5.

8 Ryan PJ, Hilton MF, Boldy DAR, et al. Validation of British Thoracic Society guidelines for the diagnosis of the sleep apnoea/hypopnea syndrome: can polysomnography be avoided? Thorax 1995;50:972-5.

9 Lévy P, Pépin JL, Deschaux-Blanc C, et al. Accuracy of oximetry for detection of respiratory disturbances in sleep apnea syndrome. Chest 1996;109:395-9.

10 Epstein LJ, Dorlac GR. Cost-effectiveness analysis of nocturnal oximetry as a method of screening for sleep apnea-hypopnea syndrome. Chest 1998;113:97-103.

11 Chiner E, Blanquer J, Arriero JM, et al. Síndrome de apnea obstructiva del sueño en la Comunidad Valenciana: situación actual, estudio de necesidades y perspectivas de futuro. Arch Bronconeumol 1998;34:177-83.

12 Johns MW. Daytime sleepiness, snoring, and obstructive sleep apnea. The Epworth sleepiness scale. Chest 1993;103: sleep

13 Rechtschaffen A, Kales A. A manual of standardised terminology, techniques and scoring system for sleep stages of human subjects. Washington DC: National Institutes of Health, Publication No. 204, 1968.

14 Gould GA, Whyte KF, Rhind GB, et al. The sleep hypopnea syndrome. Am Rev Respir Dis 1988;137:895-8.

15 Siafakas NM, Vermeire P, Pride NB, et al. Optimal assessment and management of chronic obstructive pulmonary disease. The European Respiratory Society Task Force. Eur Respir 7 1995;8:1398-420.

16 Farré R, Montserrat JM, Ballester E, et al. Importance of the pulse oximeter averaging time when measuring oxygen pulse oximeter averaging time when measuring

17 Pépin JL, Lévy P, Lepaulle B, et al. Does oximetry contribute to the detection of apneic events? Mathematical processing of the $\mathrm{SaO}_{2}$ signal. Chest 1991;99:1151-7.

18 Sériès F, Marc I, Cormier Y, et al. Utility of nocturnal home oximetry for case finding in patients with suspected sleep apnea hypopnea syndrome. Ann Intern Med 1993;119:44953.

19 Gyulay S, Olson LG, Hensley MJ, et al. A comparison of clinical assessment and home oximetry in the diagnosis of obstructive sleep apnea. Am Rev Respir Dis 1993:147:50-3. during, Brooks LJ. Normal oxyhemoglobin saturation during sleep. How low does it go? Chest 1996;110:1489-92. Douglas NJ, Thomas S, Jan MA. Clinical value of
polysomnography. Lancet 1992;339:347-50. 